\title{
Re: Dorsal Onlay Buccal Mucosal Graft Urethroplasty in Long Anterior Urethral Stricture
}

\author{
Biswajit Datta, M. P. Rao, R. L. Acharya, N. Goel, Vaibhav Saxena, S. Trivedi, U. \\ S. Dwivedi, P. B. Singh \\ Department of Urology, Institute of Medical Sciences, Banaras Hindu University, \\ Varanasi, India
}

Int Braz J Urol, 33: 181-187, 2007

To the Editor:

In this review of 43 patients with long anterior urethral stricture, the authors evaluated urethral reconstruction using dorsal onlay buccal mucosa graft with very high success rate.

I agree that ventral graft revascularization seems less reliable then dorsally securing a graft with quilting stitches to the corpora cavernosa. From a personal experience, dorsal onlay provides better visualization and less bleeding, especially for bulbar urethral part.

However, there are some issues that should be point out. It is unusual that idiopathic stenosis was the most frequent cause of stricture in their series; I suppose that most of these are uncovered lichen sclerosis and infectious etiology. I think that "simple technique" could be applied before for ventral grafting; dorsal onlay grafting, especially in very long strictures is a very sophisticated method, which requires great experience and dexterity to ensure successful outcome without serious complications. Despite great experience, the authors mentioned the need for blood transfusion in two patients, which proves that in the hands of less experienced surgeons this can be a very dangerous procedure. The authors mentioned 16 cases of panurethral strictures and the stricture length ranged from $3-9 \mathrm{~cm}$; I wonder are these cases of very short penises or the stricture did not involve whole anterior urethra. In panurethral stenoses, the graft should be as long as the penis in erect state, otherwise, postoperative ventral penile curvature as well as penile shrinkage could occur. Thus, inlaying should be in stretched or even better in erect penis.

Normal urethral stricture limits, which are determined during surgical reconstruction based on macroscopic aspect only, are not always sufficient to determine normal urethral part. Microscopic studies on presumed healthy urethral ends showed structural changes, fibrosis, which is probably the cause of residual anastomotic stenoses.

Also, we use postoperative suprapubic urinary drainage in all of our patients. Repaired urethral part is stenting by $10 \mathrm{~F}$ fenestrated stent for 7 days for two reasons: postoperative graft wetting as well as to enable evacuation of sperm in young patients due to nocturnal ejaculations. Since the sperm is the main reason for infection, we advise to all of our patients to void once after ejaculation in order to clean urethra.

Despite these few criticism and comments, I nevertheless congratulate the authors for their experience with dorsal onlay buccal mucosa graft urethroplasty in long anterior urethral strictures.

\author{
Dr. Sava V. Perovic \\ Department of Urology \\ University Children's Hospital \\ Belgrade, Serbia \& Montenegro \\ E-mail:perovics@eunet.yu
}

